\title{
Social Media Use Among Youth and Innovations of Moral Education Work With University Students in China ${ }^{*}$
}

\author{
ZHANG Wei-wei \\ China Youth University of Political Studies, Beijing, China
}

\begin{abstract}
The new media especially the use of mobile phone for information has a profound impact on contemporary university students' behavior, psychology, and way of thinking. This article will elaborate on the moral education work with university students to explore innovative ideas and innovative approaches from the perspective of interpersonal communication, political science, and sociology under the new media environment. Then the article will put forward countermeasures and improvement strategies.
\end{abstract}

Keywords: interpersonal communication, social media, innovation, moral education, university students

\section{Introduction}

Internet access and social media use have a strong radiation and driving properties with a strong diffusion effect, and the university students are with no exception. Under the new media environment, this research study to explore innovative ideas and methods of the moral education work with university students is of great significance.

Global media landscape in the 21 st century is characterized by multi-vocal, multimedia, multi-dimensional flows of information and communication. In today's digitally connected globe, flows of all kinds of information circulate around the world at a speed unimaginable even a decade ago.

New media is a 21 st century catchall term used to define all that is related to the Internet and the interplay between technology, images, and sound. In fact, the definition of new media changes daily, and will continue to do so. New media evolves and morphs continuously. What it will be tomorrow is virtually unpredictable for most of us, but we do know that it will continue to evolve in fast and furious ways. ${ }^{1}$

The advent of new media age places modern moral education of university students in China facing new situations and opportunities, as well as challenges. The article explores and analyzes how to do better in the moral education work with university students in the current new media environment by applying the key components of Harold Dwight Lasswell's communication (5W) model in the era of new media.

Access to information for university students has become diverse and complex, which makes moral education work practitioners in universities face more challenges. Therefore, the practitioners of moral education work should change the methods and systems through the establishment of multi-linked working

\footnotetext{
* Acknowledgements: This project titled "New Media and Innovative Strategies of Moral Education Work With University Students" was sponsored by "2015 Party Construction and Moral Education Work Research Fund" of China Youth University of Political Studies.

ZHANG Wei-wei, lecturer, doctor, School of Journalism and Communication, China Youth University of Political Studies.

${ }^{1}$ See http://www.newmedia.org/what-is-new-media.html.
} 
path to welcome the new era of social media and carry out effective measures.

From the point of view of social environment, economic globalization and world multi-polarization trend intensifies, and the process of China's economic and social change speeds up. Environmental changes demand for information communication between different groups become more urgent. International media power, technological power, and cultural power have also been added to this by various means into this competitive environment. Changes in the information communication market increase the dissemination of information development complexity and thus have an influence on the university students' behavior, psychology, and way of thinking. University students are aware of the access to information from the past passive acceptance to active search. The more time spent in cyberspace, the less interpersonal communication the university students have in daily communication.

Interpersonal communication is the exchange of information between individuals, including both the expressions and reactions. The main problem in this regard that many students may have is that they express only for their own gratification, react only for their own experience without much considering of others' feelings. In contrast, this article emphasizes the tendency of others (other orientation), expressions to take into account other people's reactions, reactions to understand the experience of others, that is to say, empathy.

According to Oxford Dictionary, the origin of the word "empathy" is from Greek empatheia (from em- "in" + pathos "feeling") translating German Einfühlung, first known to be used in $1850 .^{2}$ In Webster Merriam Dictionary, the full definition of empathy is presented in two ways: One is the imaginative projection of a subjective state into an object so that the object appears to be infused with it. The other is the action of understanding, being aware of, being sensitive to, and vicariously experiencing the feelings, thoughts, and experience of another of either the past or present without having the feelings, thoughts, and experience fully communicated in an objectively explicit manner; also: the capacity for this. ${ }^{3}$

American scholar Joseph A. Devito simply defined interpersonal communication as: verbal and nonverbal interaction between two persons or more. The individuals of the interpersonal communication not only mutually link, but also are interdependent, where interdependence here means: A person's behavior will have an impact on another person, a person's behavior will cause another person's behavior (Devito, 2011).

Chinese President XI Jin-ping had talked with young people before he delivered a speech at the China Academy of Space Technology on May 4, 2013, in Beijing. President XI Jin-ping encouraged young Chinese people to dare to dream, work assiduously to fulfill the dreams and contribute to the revitalization of the nation. He said the young generation with firm will, strong sense of responsibility, and great professional competence is the hope of realizing the "Chinese dream". 4

University students are the future of the country, their political awareness, political attitudes, and political participation patterns affect the future of political stability, and this study on innovation strategies of the moral education work with university students has a lasting significance. Therefore, the moral education work with the university students should keep pace with the increase of the university students' media literacy level and create innovative methods in dealing with students' affairs.

To do better work, first and foremost it is necessary for practitioners of moral education work with university students to improve the level of their media literacy and have a much deeper understanding of the

\footnotetext{
${ }^{2}$ See http://www.oxforddictionaries.com/us/definition/american_english/empathy.

3 See http://www.merriam-webster.com/dictionary/empathy.

${ }^{4}$ See http://www.chinadaily.com.cn/china/2013-05/04/content_16476313.htm.
} 
characteristics of new media communication and literacy among youth in China.

\section{Internet Access and Social Media Use Among Youth in China}

Herbert Marshall McLuhan (July 21, 1911-December 31, 1980) was a Canadian professor of English, a philosopher of communication theory, and a public intellectual. His work is viewed as one of the cornerstones of the study of media theory, as well as having practical applications in the advertising and television industries. Marshall McLuhan ${ }^{5}$ coined the phrase "global village" popularized in his books The Gutenberg Galaxy: The Making of Typographic Man (1962) and Understanding Media (1964). McLuhan described how the globe has been contracted into a village by electric technology and the instantaneous movement of information from every quarter to every point at the same time. ${ }^{6}$

Recent studies conducted by China Internet Network Information Center (CNNIC) have released The 37th Statistical Report on Internet Development in China ("Report" in short in this article) (January 2016). First, as of December 2015, China had 688 million Internet users, with a yearly increase of 39.51 million. The Internet penetration rate reached $50.3 \%$, up 2.4 percentage points from the end of $2014 .^{8}$

Secondly, from the perspective of age structure of Chinese Internet Users (see Figure 1), by December $2015,75.1 \%$ of Chinese Internet users aged between 10 to 39, and $29.9 \% 20$ to 29 . As most of the university students rang in the 20-29 age group, and according to Figure 1, nearly $30 \%$ of them use Internet. ${ }^{9}$ From the statistics quoted here, it is necessary for the moral education working staff to attach importance to the Internet access and use of university students.

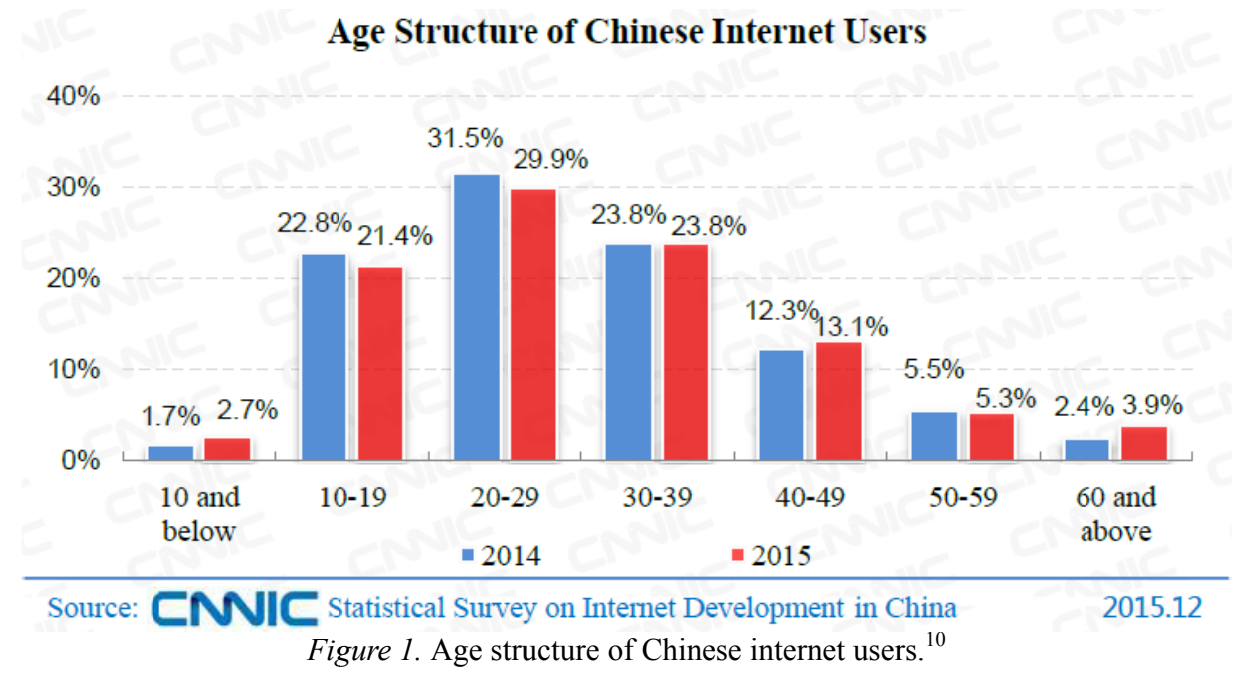

\footnotetext{
${ }^{5}$ See https://en.wikipedia.org/wiki/Marshall_McLuhan.

${ }^{6}$ See https://en.wikipedia.org/wiki/Global_village_(term).

${ }^{7}$ In 1997, state competent departments authorized to let China Internet Network Information Center (CNNIC) organize relevant Internet entities to jointly carry out the Internet development survey. Ever since then, CNNIC has published 36 statistical reports on Internet development in China, and this report is the 37th. CNNIC's reports witness the history of the Internet in China, from its infancy to takeoff, provide prudent and objective data as key references for government departments and businesses to grasp the latest development of Internet in the country and make related decisions, and thus receive extensive attention and are widely quoted at home and abroad (see source from: http://wwwl.cnnic.cn/IDR/).

${ }^{8}$ See http://www1.cnnic.cn/IDR/.

${ }^{9}$ See http://www1.cnnic.cn/IDR/.

${ }^{10}$ See http://www1.cnnic.cn/IDR/.
} 
As is shown in an article titled Youth and the (Potential) Power of Social Media written by Sheila Allison, it is possible to see that this rapid, worldwide embrace of new technology has produced a generation of young people - the "digital natives" aged 13 to 30 -who share a common culture of communication with their generational peers across borders and across continents, a "macro culture", the potential of which is yet to be fully appreciated or even acknowledged. ${ }^{11}$

Have a look at that of the US's social media use among youth, according to the Pew Research Center's ${ }^{12}$ report titled Social Media Usage: 2005-2015, which was publicized on its official website on October 5, 2015. Data from Pew Research Center shows that age has a strong association with social media usage: Those ages 18 to 29 have always been the most likely users of social media by a considerable margin. Today, $90 \%$ of young adults use social media, compared with $12 \%$ in 2005 , a 78 percentage points' increase. ${ }^{13}$

It is obvious that the high proportion use of Internet and social media among youth is a worldwide phenomenon. It is inevitable for the times that the working staff of moral education with university students should have innovative strategies and methods.

\section{Social Media Use and Moral Education With University Students}

In this part, the author addresses the question to what extent the youth use social media as their key information input sources, the impact of social media on youth in China, and in next part the author will discuss under what conditions can the functions of social media be appropriately applied to moral education work with university students, that is to say, the innovative strategies that moral education practitioners in higher education institutions will and may apply.

With adolescent Internet use has led to a host of new research questions around issues of interpersonal connectivity and teen relationships. There is a growing body of theoretical and empirical work addressing issues of how adolescents in the general population are impacted by Internet use (Rice \& Barman-Adhikari, 2014).

Adolescents in the general populace usually use the Internet to communicate, establish, and maintain relationships, and find information on a variety of issues, and for recreational and entertainment purposes (Pew Internet and American Life Project, 2009). They may also use the Internet for sociability (Rice \& Barman-Adhikari, 2014).

Young teachers in higher education institutions and college students are close of their age range, and similar level of education, environmental factors, family upbringing, and life experience make them have relative integration values. Young university teachers can give a full play to the unique advantages in the leading role model in ideological and political work (JIANG, 2014).

Correct values education is not only confined to the ideological content of education, but rather lays the basis on emotional touch, life experience understanding, and knowledge education. The communication process of the values should rely on the revealing of the relevant truth, the shock of the soul, and inner resonance. However, all knowledge, emotion, and sentiment eventually return and serve the values. The successful

\footnotetext{
11 Allison, S. (2013). Youth Studies Australia, 32(3), 69.

12 Pew Research Center is a nonpartisan fact tank that informs the public about the issues, attitudes and trends shaping America and the world. It conducts public opinion polling, demographic research, media content analysis and other empirical social science research. Pew Research Center does not take policy positions (source from: http://www.pewinternet.org/2015/10/08/social-networking-usage-2005-2015/).

13 See http://www.pewinternet.org/2015/10/08/social-networking-usage-2005-2015/.
} 
ideological and political work of university students should be the organic unity of the ideological content and the informative quality of professional knowledge, and should be the perfect combination of knowledge, feeling, and significance (JIANG, 2014).

\section{Innovations of Moral Education Work With University Students by Applying Lasswell's Communication (5W) Model in New Era of Media}

In this part, the author will apply the main components of Harold Dwight Lasswell's communication (5W) model (see Figure 2) to explain the innovations of the moral education work with university students in China. Harold Dwight Lasswell is a well-known American political scientist, who states that a convenient way to describe an act of communication is to answer the following questions. ${ }^{14}$ With the development of communication theory, the disadvantage of Lasswell's (5W) model is obvious that it is a linear model, and the noise factor and feedback are not taken into account. However, Lasswell's (5W) model does have its merits in certain circumstances. The five main components of Lasswell's communication theory indicate the key factors that will play important roles in the effectiveness of interpersonal communication between university students.

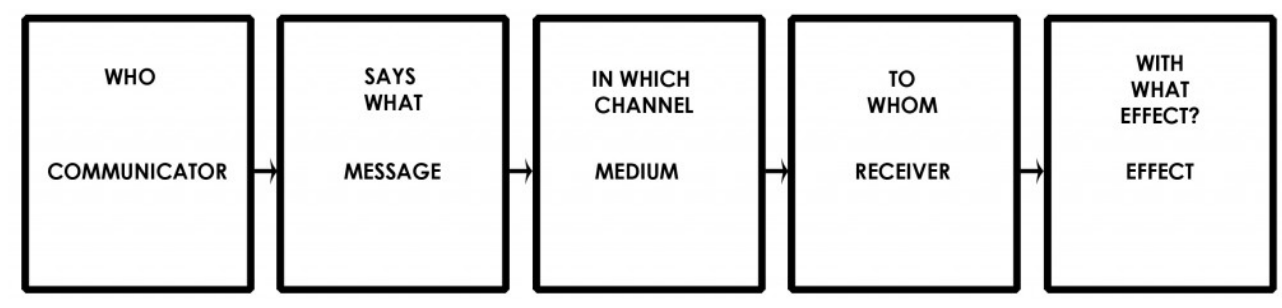

Figure 2. Lasswell's communication model (5W model).

In the following paragraphs, the author will elaborate on the research topic from the above-mentioned $5 \mathrm{~W}$ model perspective, not confined to the liner Lasswell's communication model:

Firstly, WHO perspective (communicator): refers to the working staff (practitioners) of moral education with university students here. Although in Lasswell's (5W) model, WHO, the communicator is at the beginning of the communication process. In modern communication model, the communicator, here in this article, the working staff of moral education, is in the process of circulation of communication. The practitioners of moral education work are not only responsible for information release orientated at the university students, but also receive the feedback from their audiences, the university students. Therefore, there are relatively high media literacy requirements for the practitioners of moral education work in the era of new media. Understanding communication process is also critical to the management of moral education work with university students. Communication is rarely understood as it should be. The distortion of the message can happen at any of the stages in communication process-sender, receiver, encoding, decoding, channel, message, and feedback. ${ }^{15}$

The sender is a term used in interpersonal communication, as that of communicator used in Lasswell's communication model, and here in this article it refers to the practitioners who conduct moral education work with university students in higher education institutions in China. The receiver refers to the audiences, the university students in this case. Message (information) is sent by the sender and the receiver receives the message and responds to message (feedback).

\footnotetext{
${ }_{15}$ See http://communicationtheory.org/lasswells-model/.

15 See http://ezinearticles.com/?expert=Dr.Appalayya_Meesala.
} 
Practitioners of moral education work should have the following five characteristics to be competent communicators:

(1) Responsible: This means that they take responsibility for their own communication choices and behavior;

(2) They are informed and are able to support what they say with facts and examples that are true;

(3) They are logical with developed reasoning skills and the ability to draw conclusions and reach decisions;

(4) They are accountably taking responsibility for their information, decisions, and actions;

(5) They are reliable which means they can be trusted to keep their word even if a decision may not benefit them;

(6) Accessible: They tend to value positive relationships with peers, supervisors, and clients. They are open and approachable. They are seen as caring, likable, and pleasant to be around.

Secondly, SAYS WHAT (message) perspective: Communication is complex for several reasons. Communication is interactive, symbolic, personal, cultural, irreversible, circular, purposeful, and unique.

The function of communication is to create joint meanings and shared understanding, coordinate behavior, and exchange information. Information/Message is the common basis of human relationships, understanding, and feeling.

Message is the core idea underlying communication. Creating and using symbol systems that convey information and meaning through messages. The process of human beings sharing messages is, whether for information, persuasion, or entertainment, whether in verbal or visual form, whether intentional or unintentional.

As social media and Internet access is frequently used among university students in China, therefore, it is of great importance for the practitioners doing moral education work to know that what kind of information through which channel in which format really matters. The practitioners should bear in mind: Is communication what a person says (or thought he/she said)? Or is it what the listener hears (or thought he/she heard)? How information is interpreted to create meaning for an individual student is also influenced by his/her unique make-up and background.

Thirdly, IN WHICH CHANNEL perspective: Channel can be broadly classified into two categories, verbal and nonverbal. In this article, the author focuses on the verbal channel, which includes written channel, like letters, memos, proposals, reports, presentations, e-mails, SMS texts, instant messages, social media so on and so forth, and spoken channel, like telephone conversations, video conferences, etc.

As for the practitioners doing moral education work, using multi-media platform to conduct university students' management duties is compulsory.

In new media era, no matter what channel is applied in information transmission, the messages are publicly transmitted and directed toward large, heterogeneous, anonymous audiences. The audiences are of a quantity or aggregate of considerable size. Also the message is received simultaneous to transmission and by multiple audience members.

Fourthly, TO WHOM (receiver/audience) perspective: Providing feedback is necessary for the university students in order to be offered better services. Prior knowledge can influence the receiver's understanding of the message. Verbal communication can create confusion. Every person's thought process influences the individual understanding. Feedback has to be immediate (preferably), and is much more effective if done 
face-to-face. The feedback uses media like the Internet, phone calls, letters, and so on is less effective due to delay time. The feedback should be genuine, honest, and reliable.

Fifthly, WITH WHAT EFFECT (effect) perspective: Media effects are terms used in media studies, psychology, communication theory, and sociology referring to mass media and media culture effects on individual or audience thought, attitudes, and behavior. Whether that media message has an effect on any of its audience members is contingent on many factors, including audience demographics and psychological characteristics. ${ }^{16}$ Media effects studies is complex and this article will not elaborate on it here and will discuss it later in a particular article.

\section{Conclusion}

This article will take significant steps toward the development and application of communication theory into the moral education work with university students in China. More expectation will be laid on the advancement of the understanding of media literacy in other aspects as well.

Increase the practitioners own media literacy ability to understand and make productive use of the media in their understanding of mass communication processes and industries awareness of influences on media and, in return, awareness of their influences on the university students individually and culturally.

As a starting point of social media use among youth in China from different perspectives, this article provides a moral general framework and mechanism of how to have innovations of moral education work towards university students. This research studies the characteristics of new communication model of new media varieties among university students and can deepen the understanding of the moral education work with university students under the new media environment.

\section{References}

Devito, J. A. (2011). The interpersonal communication book (12th ed., p. 7). Beijing: Renmin University of China Press.

JIANG, Y. S. (2014). Construct the collaborative ideological working model between young teachers and university students. China Higher Education, 11, 45.

Rice, E., \& Barman-Adhikari, A. (2014). Internet and social media use as a resource among homeless youth. Journal of Computer-Mediated Communication, 19, 232, 234.

${ }^{16}$ See https://en.wikipedia.org/wiki/Media_influence. 